\title{
Study on Application of Computer Vision Technology to Pneumatic Proportional Position System
}

\author{
Li Dong, Zheng Liang, Meng Zhang, Jing Yu \\ Shandong Medical College,Jinan,Shandong 250002, China \\ huner2011@foxmail.com
}

\begin{abstract}
Keywords: Computer vision, Image processing, Location, Camera calibration, Pneumatic proportional position system.
\end{abstract}

\begin{abstract}
The paper combines computer vision technology and pneumatics, and designs pneumatic proportional position system based on computer vision technology. In order to realize automatic location of pneumatic system actuator, the paper studies preprocessing technique of image including gray scale conversion of image, image enhancement, smooth, improving image quality and highlighting objective features. And the paper researches image segmentation approach, and applies mathematical morphology to process binary images, which makes image quality better.
\end{abstract}

\section{Introduction}

In recent years, with the development of computer technology, especially the development of multi media, digital image preprocess and analysis theory, and rapid development and application of large-scale integrated circuit, computer vision technology is widely applied and studied.

This paper applies computer vision technology to study the location of objects, which provides the position information of the objects for pneumatic position system, for achieving automatic location of pneumatics, which not only makes pneumatic system have object recognition ability, but also constructs the system of object automatic recognition and location. The paper realizes the application of computer vision technology to pneumatic system, constructs a computer vision pneumatic positioning system, and realizes the function of object recognition and location, which provides reference for real production application. Above al, studying computer vision, especially studying the application of it to industry, has great importance to improve production technology and develop industry.

\section{Image gray processing}

In order to accelerate the processing speed of images, the acquired color images need to be converted into gray images, which is called gray processing. It makes R, G and B of RGB model equal. Gray processing is the process that color images with light and color are converted into gray images. The graying result is the basis of processing the subsequent images.

In general, each pixel of color image is represented by three bytes. Each byte corresponds to the light (red, green and blue) of R, G and B component. The pixel of the black-and-white image after conversion uses one byte to represent the gray value which is between 0 and 255 . The greater the value is, the whiter the point is. The transformational relation is:

$$
\operatorname{Gray}(i, j)=0.299 R(i, j)+0.587 G(i, j)+0.114 B(i, j)
$$

$\operatorname{Gray}(i, j)$ is the gray value of black-and-white image after transformation on point $(i, j)$

In formula (1), green has the largest proportion, so $G$ value can be used to be as the gray after transformation.

Gray processing has the other methods such as taking the maximum, minimum and average value of three components. The purpose is to make the values of $\mathrm{R}, \mathrm{G}$ and $\mathrm{B}$ component equal.

For gray-scale images, each pixel value can be represented by one byte or eight bits, which can show $256\left(2^{8}\right)$ levels. It means that gray-scale images only can show 256 colors, and the gray-scale images only have gray level and have no color. 


\section{Image Segmentation and Location}

After the image receives gray-scale conversion, histogram equalization and smooth, the image is segmented. The objects of the image are separated from the background, which forms a binary image. Based on the image, morphological processing is made to make the centroid coordinates calculation accurate, which realizes pixel coordinates location of object image centroid.

Segmentation based on regions. Selecting gray threshold for binarization processing on object image is one of the most typical, simple and effective image segmentation methods. Before taking out geometric features of gray scale images, the images must receive threshold processing, which means the process of taking out the part above or below the gray value. An acquired image includes object, background and noise. The most common method for how to take out the required object image from many-valued images is to suppose a threshold value $t$, and t divides the data of the image into two parts, the pixel which is greater than $t$ and the pixel which is less than $t$. If the input image is gray scale image $f(i, j)$, and the output image is $g(i, j)$,

$$
g(i, j)= \begin{cases}1, & f(i, j) \geq t \\ 0, & f(i, j)<t\end{cases}
$$

If the method of gray scale transformation is used to study the image $f(i, j)$, the method is called binarization. Binarization processing means that the image $f(i, j)$ is divided into object and background according to the threshold value.

Two threshold values can be used to take out the small range of gray scale of the object image contour as 1 , which makes the outline of the object clear. The output image $g(i, j)$ is represented as follows.

$$
g(i, j)= \begin{cases}1, & t_{1} \leq f(i, j) \geq t_{2} \\ 0, & f(i, j)<t_{1} \text { and } f(i, j)>t_{2}\end{cases}
$$

If the gray scale transformation function is 0 when it is less than a gray value, the above conditions keep invariable,

$$
g(i, j)=\left\{\begin{array}{l}
f(i, j), f(i, j) \geq t \\
0, \quad f(i, j)<t
\end{array}\right.
$$

The processing method is called semi-threshold processing. When gray value of the background is compared with the object, it has significance that the gray value is very low.

The image in the paper is the gray scale image after processing, so the binarization formula is $g(i, j)=\left\{\begin{array}{l}255, f(i, j) \geq t \\ 0, f(i, j)<t\end{array}\right.$

The background of the image is 255(white), and the object is 0 (black).

Selecting appropriate threshold is very important for segmentation based on regions. Determining an appropriate threshold can separate the object image from the background image. Therefore, selecting threshold plays a critical role in accuracy of segmentation of the image and figure center calculation of object image.

Determination of threshold. The paper uses iterative method to determine the threshold, and the following is the procedure.

Firstly, the maximal and minimal gray value of the image, $Z_{1}$ and $Z_{k}$, are figured out, and the initial threshold is

$$
T^{k}=\frac{Z_{1}+Z_{k}}{2}
$$

According to the threshold $T^{k}$, the image is segmented into object image and background image. And the average gray valu $Z_{O}$ and $Z_{B}$ of two parts are figured out. 


$$
\begin{aligned}
& Z_{o}=\frac{\sum_{Z(i, j)<T^{k}} Z(i, j) \times N(i, j)}{\sum_{Z(i, j)<T^{k}} N(i, j)} \\
& Z_{B}=\frac{\sum_{Z(i, j)>T^{k}} Z(i, j) \times N(i, j)}{\sum_{Z(i, j)>T^{k}} N(i, j)}
\end{aligned}
$$

In the formula, $Z(i, j)$ is the gray value of of the image, and $N(i, j)$ is the weighting coefficient of $(i, j)$ and is 1 .

$$
T^{k+1}=\frac{Z_{O}+Z_{B}}{2}
$$

If $T^{k}=T^{k+1}$, the iteration ends, or $k \leftarrow k+1$, and it turns to the iteration of the second part.

The threshold based on iteration can differentiate the foreground and background of the image, but it can't differentiate subtlety of the image. For some specific images, the change of small data may make segmentation effect greatly different.

Image segmentation effect. The above method is used to make segmentation process on the acquired image. The left figure of Figure 1 is the original data, and the right figure is the effect figure after segmentation process. We can see from the figure that the object completely separates from the background, which forms a binary image that the background is white and the object is black.

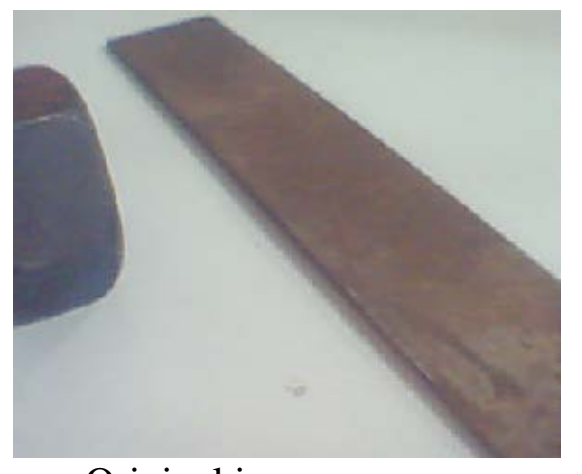

Original image

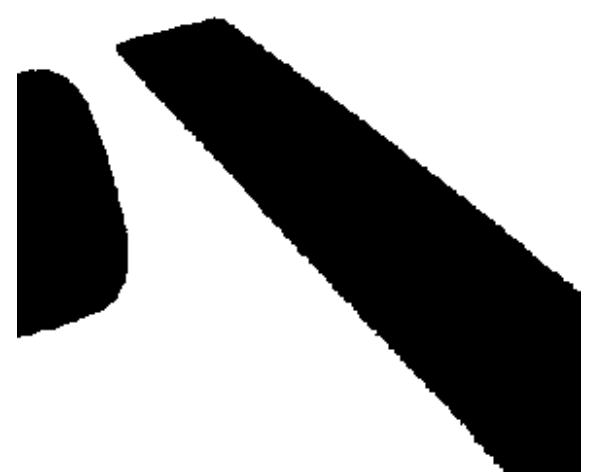

Image after segmentation

Figure 1 Segmentation effect

\section{Computer Vision Positioning System composition}

Object image processing and locating experiment. From the computer calibration experiment and the internal and external parameters of the camera, we can see that the transformation between coordinate systems can make pixel coordinate value of the image achieve the plane coordinate of the objects on real work platform.

The nuts are placed in turn. The achieved data from measurement, the data after transformation and the practical measured data are shown in Table 1.

We can see from the data in Table 1 that there is no great difference between the position data achieved by computer vision and the real position data of the object, and computer vision can figure out the location of the object, which meets the requirements of pneumatic proportional position system based on computer vision.

Cylinder positioning experiment. The paper mainly discusses the location of experiment cylinder. The output voltage of the proportional valve is 5.7 and $4.8 \mathrm{~V}$, which makes the cylinder move at low speed. The pneumatic system receives locating experiment according to the program by pneumatic system control module. And the experiment data is shown in Table 2. 
Table 1 Locating data and processing

\begin{tabular}{|c|c|c|c|c|c|c|}
\hline & \multicolumn{2}{|c|}{$\begin{array}{l}\text { Pixel coordinate } \\
\text { (pixel) }\end{array}$} & \multicolumn{2}{|c|}{$\begin{array}{l}\text { Measured coordinate } \\
(\mathrm{mm})\end{array}$} & \multicolumn{2}{|c|}{$\begin{array}{l}\text { Practical coordinate } \\
(\mathrm{mm})\end{array}$} \\
\hline & $\mathrm{U}$ & $\mathrm{V}$ & $\mathrm{X}$ & $\mathrm{y}$ & $x_{\omega}$ & $y_{\omega}$ \\
\hline 1 & 123 & 95 & 10.091 & 8.335 & 10.000 & 10.000 \\
\hline 2 & 154 & 96 & 30.992 & 9.042 & 30.000 & 10.000 \\
\hline 3 & 185 & 98 & 50.844 & 8.964 & 50.000 & 10.000 \\
\hline 4 & 216 & 99 & 70.696 & 8.883 & 70.000 & 10.000 \\
\hline 5 & 246 & 100 & 90.543 & 8.804 & 90.000 & 10.000 \\
\hline 6 & 276 & 101 & 110.054 & 8.692 & 110.000 & 10.000 \\
\hline 7 & 307 & 102 & 130.561 & 8.587 & 130.000 & 10.000 \\
\hline 8 & 337 & 103 & 150.412 & 8.502 & 150.000 & 10.000 \\
\hline 9 & 368 & 104 & 170.267 & 8.421 & 170.000 & 10.000 \\
\hline \multicolumn{7}{|c|}{ Table 2 Cylinder locating data } \\
\hline & \multicolumn{2}{|c|}{$\begin{array}{ll}\begin{array}{l}\text { Destination location } \\
(\mathrm{mm})\end{array} & \\
\end{array}$} & \multicolumn{2}{|c|}{$\begin{array}{l}\text { Actual locating } \\
\text { position (mm) }\end{array}$} & \multicolumn{2}{|c|}{$\begin{array}{l}\text { Positional accuracy } \\
(\mathrm{mm})\end{array}$} \\
\hline 1 & \multicolumn{2}{|c|}{10.000} & \multicolumn{2}{|c|}{10.034} & \multicolumn{2}{|l|}{0.034} \\
\hline 2 & \multicolumn{2}{|l|}{30.000} & \multicolumn{2}{|l|}{30.050} & \multicolumn{2}{|l|}{0.050} \\
\hline 3 & \multicolumn{2}{|l|}{50.000} & \multicolumn{2}{|l|}{50.031} & \multicolumn{2}{|l|}{0.031} \\
\hline 4 & \multicolumn{2}{|l|}{70.000} & \multicolumn{2}{|l|}{70.042} & \multicolumn{2}{|l|}{0.042} \\
\hline 5 & \multicolumn{2}{|l|}{90.000} & \multicolumn{2}{|l|}{90.040} & \multicolumn{2}{|l|}{0.040} \\
\hline 6 & \multicolumn{2}{|l|}{110.000} & \multicolumn{2}{|l|}{110.051} & \multicolumn{2}{|l|}{0.051} \\
\hline 7 & \multicolumn{2}{|l|}{130.000} & \multicolumn{2}{|l|}{130.032} & \multicolumn{2}{|l|}{0.032} \\
\hline 8 & \multicolumn{2}{|l|}{150.000} & \multicolumn{2}{|l|}{150.042} & \multicolumn{2}{|l|}{0.042} \\
\hline 9 & \multicolumn{2}{|l|}{170.000} & \multicolumn{2}{|l|}{170.033} & \multicolumn{2}{|l|}{0.033} \\
\hline
\end{tabular}

\section{Summary}

The paper uses camera as image acquisition equipment, designs pneumatic positioning system based on computer vision, combines computer vision technology and pneumatic proportional control technology, and realizes determining the position of object by computer program and realizes automatic location of pneumatic system. And the paper makes experiment for verification.

\section{References}

[1] Behrenbrush Christian $P$ et al. Fusion of contrast-enhanced breast MR and mammographic imaging data. Medical Image Analysis, 2003, 7(3):311-340.

[2] Welsh Alec W et al. Development of three-dimensional power Doppler ultrasound imaging of fetoplacental vasculature. Ultrasound in Medicine and Biology, 2001, 27(9):1161-1170.

[3] P.K. Allen, B. Yoshimi, A. Timcenko. Real-time Viaual Servoing. In Proc. Of IEEE Conference on Robotics and Automation.1992: pp1850-1856.

[4] W.Jang, Zbien. Feature-based Visual Servoing of an Eye in hand Robot with Improved Tracking Performance. In Proc. IEEE Int. Conf. Robotics and Automation. 1991:pp2254-2260.

[5] Serra J. Image Analysis and Mathematical Morphology. London: Academic Press, 1982.

[6] Chesi Graziano, Hasimoto Koichi. A self-calibrating technique for visual servoing. Proceedings of the 41st IEEE Conference on Decision and Control, 2002:2878-2883.

[7] B Caprile, V Torre. Using Vanishing Points for Camer Calibration. Int. Journal of Computer Vision, 1990, (4):127-140.

[8] Faugeras O. D, Luong Q T, S J. Cameraself-calibration: Therory and experiments. In: Proceedings of the European Conference on computer Vision. Italy: Santa-Margerita, 1992. 